\title{
SCIENCE
}

Editorial Committee: S. Newcomb, Mathematics; R. S. Woodward, Mechanics ; E. C. Pickering, Astronomy; T. C. Mendenhall, Physics; R. H. Thurston, Engineering; Ira Remsen, Chemistry;

J. Le Conte, Geology; W. M. DAvis, Physiography; O. C. MARsh, Paleontology; W. K.

Brooks, C. Hart Merriam, Zoölogy; S. H. Scudder, Entomology ; N. L. BritTon,

Botany; Henry F. Osborn, General Biology ; H. P. Bowditch, Physiology;

J. S. Billings, Hygiene ; J. McKeen Cattell, Psychology ;

Daniel G. Brinton; J. W. Powell, Anthropology ;

G. BRowN GOODE, Scientific Organization.

Friday, May 15, 1896.

CONTENTS :

Some Problems about to Confront Astronomers of the Twentieth Century: J. K. ReEs.....................717 On a New Form of Radiation : W. K. RöNTGEN..726 Behavior of Sugar towards Röntgen Rays: FERDINAND G. WIECHMANN.. .......................729

The $X$-Rays in Medicine and Surgery: CHARLES L. NoRTon........................................730

Current Notes on Physiography:-

De Lapparent's Leçons de géographie physique: The Interior Plateau of British Columbia; The Volcanic Group of Topographic Forms; Le tour du monde; Thunder Storms at Sea are Nocturnal; W. M. DAVIS.

Current Notes on Anthropology:-

The Anthropological Institute of Great Britain; Canadian Archæology: D. G. BRINTON ..........,733

Scientific Notes and News:-

Batrachians and Crustaceans from the Subterranean Waters of Texas; The Forest Resources of the United States; Cape Colony Geological Commission; The Metric System; General....

University and Educational News.......................738

Discussion and Correspondence:-

Principles of Marine Zoögeography: ARNOLD E. ORTMANN. The Child and Childhood in FolkThought: Franz Boas. The Discussion of Instinct: C. Lloyd Morgan. The Subject of Consciousness: JoHANNEs REHMkE. The Prerogatives of a State Geologist: ERASMUS HAwORTH. A Correction: T. A. JAGGAR, JR. The Absolute and the Relative: J. W. Powell..739

Scientific Literature:-

Marcou's Life, Letters and Works of Louis Agassiz. Mosso's Fear: J. MCKeEN Cattell. Hueppe's Bakteriologie: H. W. ConN.............745

Scientific Journals :-

The Astrophysical Journal; The American Geologist

Societies and Academies:-

The Academy of Science of St. Louis: WILLIAM TRELEASE. New York Academy of Sciences, Section of Anthropology, Psychology and Philology: LIVINGSTON FARRAND. Torrey Botanical Club : W. A. BASTEDo. Geological Conference of Harvard University: T. A. JAGGAR, JR. Philosophical Society of Washington: BERNARD R. GREEN.
SOME PROBLEMS ABOUT TO CONFRONT ASTRONOMERS OF THE TWENTIETH CENTURY.

Members of the New York Academy of Sciences, Ladies and Gentlemen: The nineteenth century has shown vigorous development in all branches of science, and in none more than in astronomy. The effective work of numerous observers and mathematicians has lifted us to greater heights of knowledge, making visible and clear many things previously discerned dimly. But the elevation has also extended our horizon, and the boundaries of knowledge appear 'infinitely infinite.' This evening I shall not make any attempt to sketch the details, or even the general features of the view before us, as we stand at the end of the century, looking down from the elevated position the scientific workers in astronomy enable us to occupy. I shall content myself with a much narrower survey, selecting here and there some especial part of the field before us, with the desire of stating briefly what has been done in that field and in what condition it now stands.

From the point of view of the practical astronomer the stars are so many signal lights marking the 'milestones on the great celestial highway traversed by the planets, as well as on the byways of space occasionally pursued by comets.' If we desire to know the position of a planet or a comet on

*Address of the retiring President of the New York Academy of Sciences, March 30, 1896. 
the celestial vault, we must observe how far such a body is separated in two directions from the neighboring stars, and evidently we must know where these stars are situated in the heavens. A few thousand stars are sufficient for this purpose provided they are determined in position with the highest precision.

But the astronomer is also deeply interested in 'the sublime problem of the construction of the heavens' and many thousands of stars must be exactly located to aid in solving this problem. Moreover, there is need of a general rollcall of all the stars visible in ordinary telescopes. Such a ' rollcall' or ' index' gives the positions of the stars with an accuracy less than the highest precision requires, and is mainly useful as a basis of work for the more accurate catalogues. The work of determining the positions of stars on the sky-dome is the most important and fundamental operation in practical astronomy. During the present century this foundation work of astronomy has been carried on "with a zeal and success by which all previous efforts are dwarfed into insignificance.'

The great German astronomer ' the unrivalled Bessel' from 1821 to 1833 made some 75,000 observations, by which the number of fairly well determined stars was increased to above 50,000. His assistant and successor, Argelander, who gave up finance for astronomy, using a glass only two and a half inches in diameter, recorded 324,189 stars down to the $9 \frac{1}{2}$ magnitude. This number included all the stars of the magnitude named, visible in the northern hemisphere of the heavens, and in addition a small zone about two degrees wide, south of the celestial equator. Schönfeld continued the survey at Bonn, down to the southern tropic.

In 1882 a photograph taken of the great comet of that year, by Dr. Gill at the Royal Observatory at the Cape of Good Hope, showed so many stars that it was determined to use photography in completing the Bonn survey to the south pole. The exposure of the plates in duplicate required four years from 1885-89. But this was the least laborious part of the great work. These plates had to be measured and the measurements reduced so as to obtain the proper positions of the stars on the sky. Prof. Kapteyn, of Gröningen, lately completed this task, and the catalogue from the plates is now passing through the press. The catalogue will contain about 350,000 stars to the tenth magnitude. A considerable part of the southern sky covered by the surveys of Schönfeld and Gill was examined also by Dr. Gould. Through 'unceasing labors during his fifteen years' residence a Cordoba,' in the Argentine Republic, an acquaintance of some 73,160 stars down to the $9 \frac{1}{2}$ magnitude was brought about. The Argentine General Catalogue was published in 1886 and contained the accurate places of 32,448 southern stars. These and other catalogues put us in possession of a list of stars fairly well determined in position, numbering nearly 700,000. Catalogues of much greater precision, giving the positions of a smaller number of stars with the highest accuracy, have been prepared after many years of observation and calculation by the noted observatories of the world.

As an example of coöperation in modern science, I ought to mention that the first organized effort for determining star positions with the highest precision was made by the German Astronomical Society in 1865. The scheme, now practically finished, was to fix the positions accurately of about 100,000 stars on Argelander's list and some 30,000 from Schönfeld's. Thirteen observatories were interested in this great work, each being assigned a 'zone;' two in this country-Harvard College Observatory and the Dudley Observatory at Albany. 
The work of Lewis M. Rutherfurd, of this city, an honored member of this Academy for many years, in photographing stellar clusters and in measuring the plates with a machine of his own devising, was the first serious attempt to use photography in getting the exact relations of stars to each other. The recent publications of the Columbia College Observatory show unmistakably that from these measures relative positions of the highest precision are obtainable. The invention of dry plate photography has made the photographic work of the astronomer of to-day much more expeditious, and enables him to secure many more stars on his plates with a given time of exposure. The last years of this century witness the carrying out of a gigantic plan for making an enormous catalogue of the highest precision by the aid of photography and supplementing this catalogue by a series of charts.

On April 16, 1887, there met in Paris 56 delegates of 17 different countries, to discuss ways and means of carrying out this grand photographic work. The final decision was to construct a photographic chart of the heavens of all the stars down to the 14th magnitude. On these plates will appear, it is estimated, some 20,000,000 stars. Methods are now being devised to reproduce accurately these chart plates. It was decided also to supplement these chart plates, made with an exposure of 40 minutes, by plates of shorter exposures, from measurements of which, with machines of the highest precision, a catalogue is to be prepared. These catalogue plates show the stars down to the 11th magnitude, and the number of stars may reach two millions. Twenty-two thousand plates (duplicated and overlapping) will be necessary for the catalogue. The work has been going on for several years at 18 observatories throughout the world (except in the United States) and the photographic part will soon be finished. The measurements of the plates and the calculations based thereon are also being carried on at Paris, Potsdam, Greenwich and elsewhere. Judging from the work already done, we may confidently expect that nearly all the results will be ready for printing in about ten years. The astronomers of the 20th century will then be in possession of material which will aid them in studying the problems connected with the construction of the universe of stars.

The number of stars around us increases with every augmentation in telescopic power, and in time of exposure of photographic plates. The largest telescopes will show perhaps more than $60,000,000$ stars. The long exposure photographs (say 12 hours) would show many millions more. M. l'Hermite has ' computed the population of the stellar universe from his valuation of stellar light power and finds it, on the assumption that the scattering of the stars is everywhere just as it is in our own neighborhood, to be sixty-six thousand millions!' This result is ingenious and interesting, but depends for its value on the above assumption.

The task of sidereal astronomy-a stupendous one-is this then: 'to investigate the nature, origin and relationships of millions of stars; to inquire into their movements among themselves and that of our sun among them,' and ' to assign to each its place and rank in the universal order.'

Among the great number of interesting problems in sidereal astronomy, let me select two or three of the most important. The catalogues of the highest precision enable the astronomer to determine the positions of various stars at widely different dates. This requires that the catalogues used should be made up from observations at those dates. Now comparing the positions of a star at any two dates a difference will be found depending in amount on the lapse 
of time. The star, therefore, has apparently moved from the first to the second position. But there are several things to be taken into account before we can say how much the star has moved. The effects of precession and nutation and the aberration of light must be eliminated. When this is done it is found that only a very few stars have an accurrately determined motion of their own among their fellows. Schönfeld, of Bonn, has published a list of 83 stars that have a proper motion in a year greater than $\mathcal{I}^{\prime \prime}$ of arc, $i . e$. , greater than the angle subtended by three-tenths of an inch to the eye placed one mile away. Only 83 of all the hosts of stars are known to move at right angles to our line of sight $1^{\prime \prime}$ of arc or more in one year.

The star which has the greatest proper motion so far known is No. 1830 of Groombridge's Catalogue. This star moves 7!! of arc in a year. The star is so far away that this small apparent motion across our line of sight means, if the distance has been accurately determined, a very startling linear velocity of more than 230 miles in a second, a speed ' uncontrolable, according to Newcomb, by the combined attractive power of the entire sidereal universe.'

Groombridge 1830 is not the only ' runaway' star in the list, there are several others; Clerke remarks "the fact then confronts us that not a few of the stars possess velocities transcending the power of government of the visible sidereal system. Is that system threatened with dissolution, or must we suppose the chief part of its attractive energy to reside in bodies unseen, because destitute of the faculty of luminous radiation? No answer is possible ; conjecture is futile. We are only sure that what we can feebly trace is but a part of a mighty whole, and that on every side our imperfect knowledge is compassed about by the mystery of the Infinite."

When we consider proper motions less in amount than 1" a year the list swells in number to over 3,000 . The astronomer of the XXth Century will be able to determine the proper motions of thousands of other stars, using the superb catalogues constructed in this century. In studying this problem the aid of the spectroscope has been called in, and with that wonderful instrument it has been found possible to measure the velocity of quite a number of stars in the line of sight, either directly from us or toward us. At Potsdam and at Greenwich and elsewhere, by the use of measurements or photographs of stellar spectra or by visual observations, Aldebaran, the brighest star in the constellation of Taurus, has been found to be moving from the earth at the rate of 30 miles a second. The Greenwich observers tell us that the North Star is moving toward us at the rate of 16 miles a second. Vogel, at Potsdam; places the motion of Arcturus at 45 miles a second from the earth. When we combine the motion in the line of sight with the motion at right angles to that line, we can discover the real motion in space, its amount and direction. At the Lick Observatory Mr. Campbell has proposed to determine with the spectroscope in what direction the solar system is moving among the stars.

The motion of our solar system among the stars has interested astronomers for many years. Sir Wm. Herschel first investigated this problem a century ago. The fundamental principle of the investigation is this: Those stars which lie in the direction in which we are going will appear to open out from each other, while those in the part of the sky that we are leaving will close up behind us. Since Herschel's time the materials for investigation have been greatly augmented. An examination of the stellar proper motions has been made by many calculators, and especially recently by Prof. Boss, of Albany, and by Mr. Oscar 
Stumpe, of Bonn. The results agree as well as we could expect at present in fixing 'the brilliant Vega' as 'the center round which the new determined apexes tend loosely to group themselves.' The general direction of the solar motion is thus fairly well determined. The velocity of this motion has not yet been accurately worked out. Sixteen miles a second is given by some astronomers as a probable value.

The distances of the stars have always excited the curiosity of man. During this century the refined methods for obtaining reliable values have been worked out. Only within the last twenty years have the most accurate values been determined. The solar system to our finite minds seems isolated in space, the nearest star being so far away that light traveling at the rate of 186,330 miles in each second of time consumes 4.35 years in reaching the earth. The parallax of $\alpha$ Centauri is $0 .{ }^{\prime \prime} 75, i$. e., the distance separating the earth from the sun, over 90,000,000 miles, would appear to the eye of an observer on $\alpha$ Centauri, as small as $\frac{2}{10}$ of an inch appears to our eyes at a distance of one mile. This nearest star, $\alpha$ Centauri, is at the head of a list of less than 60 stars whose parallaxes have been determined with all the accuracy, very nearly, at present possible. But the laborious search for measurable stellar parallaxes has not been extensive enough among the millions of stars to make us feel that astronomers have determined certainly even the nearest star. Perhaps it will be found among some of the fainter telescopic stars, or even on the photographic plates, that with long exposure show us stars so faint that we can never expect to see them. Photography has proved itself to be a most valuable aid in this investigation, and from the plates specially made much more is to be expected in the future.

The telescope shows numerous cases in which two stars are so close to each other that they can be separated only by a high magnifying power. These are 'double stars.' The catalogues now enumerate more than 10,000 such couples, and the number known to us is increasing quite rapidly. One of the chief pieces of work in which the largest telescopes are used is in detecting new cases of very faint and exceedingly close doubles. A careful examination has revealed the fact that some 200 or more cases of double stars show that the components are physically connected.

The components revolve about the common center of gravity of the system. When one of the stars is much greater in mass than the other, the second star, usually the fainter, revolves about the larger one. Many of these binary stars as they are called are of great interest. Their times of revolution range from 14 years to 1,500 years. The orbits are comparable with the larger orbits of the solar system, some of them being twice as large as that of the planet Neptune, which, as you will remember, moves in an orbit having a radius of about $2,800,000,000$ of miles, and revolves about our sun in 165 years nearly.

There are cases of multiple stars. Epsilon Lyrae is a beautiful quadruple star, composed of two pairs. Each pair makes a slow revolution in a period of over 200 years. It is thought that there is evidence that the two pairs revolve about the common center of gravity of the four stars.

Peters found in 1851 that the apparent irregularities in the movements of the brilliant Dog star Sirius could be fully explained by an orbital revolution in a period of fifty years. Bessel had announced in 1844 that the two bright Dog stars Procyon and Sirius moved in seeming irregular paths, because of the presence of unseen bodies near them. Peters thus vindicated Bessel's prediction. On the 31st of January, 1862, while testing the new 18-inch object glass ordered by the late Pres. Barn- 
ard for the University of Mississippi, Alvan G. Clark discovered a faint companion to Sirius. This proved to be in the exact position required by Peters' calculations. For Procyon no companion has been found. Recently there have been found evidences of an unseen body in the system of 70 Ophiuchi, a wide double. These and similar investigations indicate that there may be myriads of systems in space similar to our own. Painstaking observations and exact calculations will, no doubt, reveal many hundreds of these systems during the next century, even before new inventions have increased our seeing power.

The thoughtful observer is struck by the fact that the light of most of the stars does not appear to change; they remain each of them apparently of the same brightness year after year, and so far as we can judge from previous accounts, century after century. The stars are so far away that changes in their light-giving power are in most cases invisible to us. There are, however, now known nearly 400 stars which show a variation in light. Some stars change their brightness slowly and continuously; others fluctuate irregularly, like the wonderful star $\eta$ Argus in the southern heavens, which was nearly as bright as Sirius in 1843 and decreased in brightness down to the 7 th magnitude in 1865 . It remained at that magnitude until 1888 and has since been increasing in brightness. Dr. Gill, at the Cape of Good Hope, has been studying the star by the aid of photography during the past few years.

Then there is a class of variables called 'temporary stars.' These blaze out suddenly and then disappear. Such variables are styled, sometimes, 'new stars.' Pickering gives a list of 14 'new stars' discovered since the time of Tycho Brahe in 1572. In this list all but four belong to this century, no temporary star being recorded between 1670 and 1848. Six new stars are recorded as having been discovered since 1886. Two of these in 1895. There is no doubt that a more careful study of the heavens will reveal many more such cases. Several of the stars of this class were brightly visible to the naked eye. They remained visible with fading light for different periods of time. Future investigation may show that these stars will appear again, and thus indicate that they are variables of long period showing such light changes as to place their minima beyond the power of the telescope or even of the photographic plate. Then there are known to be a considerable number of variables whose periods of light changes are well determined. These are most interesting to observe. One class has a period of several months, another class a period which is quite short, and still another class ' in which the variation is like what might be produced if the star were periodically eclipsed by some intervening object.' Great use is now made of photography both of the stars and of their spectra in studying variables. At the Harvard College Observatory the plates taken in Arequipa, Peru, have shown on examination many variables. Pickering states that in two photographs of the cluster Messier 5 taken August 9, 1895, only two hours apart, forty-six variables of short periods were found! In the photographs of stellar spectra the presence of bright hydrogen lines in conjunction with dark lines or dusky bands has led to the discovery of numerous variables. The subject is of growing interest and the further prosecution of the work will add, no doubt, many thousands to the present list which has recently been brought down to date by Dr. S. C. Chandler, of Cambridge.

The study of the sidereal systems presents many problems for the mathematicalastronomer, but let us consider some unsolved problems connected with our own system. "The profoundest question growing out of the the- 
ory of gravitation is whether all the inequalities in the motion of the moon and the planets admit of being calculated from their mutual attraction." In order to answer the question the astronomer must make the calculations demanded by theory, giving him the positions of the planets considered, and then compare the calculated with the observed place. No complete solution has ever been found even in the case of three bodies, and for the case of a larger number of planets no approximation to an entire solution has been made. The complexity of the problem is due to the fact that "the forces which act upon the planets are dependent upon their motions, and these again are determined by the forces which act on them."

Many great mathematicians from Newton's time till now have given much of their attention to the question of how to surmount the difficulties. The success of the partial solution is attested by the "marvellous accuracy with which sun, moon and planets move in their prescribed orbits." Though the accuracy is marvellous, there are two cases of greatest interest especially demanding the attention of the mathematical astronomers. These two cases have to do with the motions of the Moon and of Mercury.

The 'Tables of the Moon,' calculated by Hansen and published in 1857 by the British government, were supposed to provide the astronomer with the means of calculating accurately the position of the moon for a century or more. Prof. Grant, in his 'History of Physical Astronomy,' published in 1852, remarked: "Thus the clouds which for a moment obscured the Newtonian theory of gravitation have been effectually dissipated, and a fresh conquest has been added to the long list of triumphs which adorn its history." The agreement of observed and calculated position from 1750 to 1850 is all that could be desired, but it has been found that previous to $\mathbf{1 7 5 0}$ and after 1850 the calculations and observations do not agree closely enough to satisfy the mathematical astronomer. Mr. Stone, of Oxford, has published a table (M. N. R. A. S., LII., No. 7, p. 478) showing the 'mean excess over observation of the moon's tabular place in longitude for the years 1847 to 1891, as computed from Hansen's tables.' It is therein shown that from 1847 to 1863 the calculated longitude differed from the observed by a mean annual value of $-1^{\prime \prime} .85$ and no law of regular change is apparent. Since 1863 the mean annual error has increased at an average rate of $0^{\prime \prime} .75$ per annum. The error now amounts to about $20^{\prime \prime}$, equal to about $\frac{1}{100}$ of the moon's angular diameter.

The lunar tables have been empirically corrected by Newcomb and also by Tisserand and at present the results are satisfactory. However, gravitation seems unable to explain theoretically the movement of the moon's perigee. The mathematical astronomer will no doubt triumph over the new obstacle which presents itself to-day, but, as Tisserand says, a beautiful discovery remains to be made.

Newcomb has stated that "another change not entirely accounted for on the theory of gravitation occurs in the motion of the planet Mercury." Leverrier fourd "that the motion of the perihelion of Mercury is about 40 " in a century greater than that computed from the gravitation of the other planets." He attributed this to the attraction of a 'group of small planets between Mercury and the sun.' Newcomb, in his recent work on 'Astronomical Constants,' gives the result of an examination of this hypothesis as well as of several others. He concludes (1) "that there can be no such non-symmetrical distribution of matter in the interior of the sun as would produce the observed effect." (2) The hypothesis of an intra-mercurial ring or group 
of planetoids seems to be untenable. (3) The hypothesis of an extended mass of diffused matter like that which reflects the zodiacal light has insurmountable difficulties.

The hypothesis of a ring of planetoids between the orbits of Mercury and Venus is very unsatisfactory.

Newcomb finally regards Prof. Hall's hypothesis as not inadmissible. This hypothesis is a startling one, no less than that gravitation toward the sun is not exactly as the inverse square of the distance. Prof. Paul Harzer has recently published a memoir dealing with this subject, which obtained the prize of the Jablonowski Society. Harzer is disposed to attribute the greater motion of Mercury's peribelion to an irregular distribution of the sun's mass within its surface, admitted to be spherical; being denser in the parts near the solar equator. He appears to think the solar corona may have something to do with it. Harzer's theory seems to have the advantage over Newcomb's modification of Newton's law in that it leaves the latter intact.

Newcomb considers that, with the exception of the motions of the moon and of Mercury, "all the motions in the solar system, as far as known, agree perfectly with the results of the theory of gravitation. The little imperfections which still exist in the astronomical tables seem to proceed mainly from errors in the data from which the mathematicians must start in computing the motion of any planet. The time of revolution of a planet, the eccentricity of its orbit, the position of its perihelion, and its place in the orbit at a given time, can none of them be computed from the theory of gravitation, but must be derived from observations alone. If the observations were absolutely perfect, results of any degree of accuracy could be obtained from them; but the imperfections of all instruments and even of the human sight itself prevents obser- vations from attaining the degree of precision sought after by the theoretical astronomer and make the consideration of ' errors of observation' as well as 'errors of the tables' constantly necessary."

One of the most important and interesting investigations going on now deals with the subject of variation of latitude. Certain theoretical considerations led the astronomers fifty years ago to look for changes of latitude which showed a period of 305 days. Maxwell and Bessel examined the matter and Bessel found that his latitude diminished $0^{\prime \prime} .3$ in two years (1842). Other observations at various places showed aparent changes of small amounts. The results for several reasons that then appeared sound were not regarded as satisfactory, so that it was doubted by many that any measurable variation of latitude would be found. The problem assumed a new aspect, however, when Dr. Küstner, of Berlin, published the results of his observations made in 1884-85. These results showed unmistakably that a small but quite a rapid change had occurred in the latitude of Berlin, amounting to from $0^{\prime \prime} .2$ to $0^{\prime \prime} .3$. The examination of other observations showed similar results.

A crucial test was made by sending an expedition to the Sandwich Islands, which is 180 degrees (nearly) in longitude from Berlin. If, it was known, the latitude of Berlin increased, then a point in the northern hemisphere, 180 degrees away from Berlin, should simultaneously show a decrease in latitude, for if the pole moves toward Berlin it must move from the point on the other side of the earth. Our own government joined in the effort. Marcuse, of Berlin, and Preston, of Washington, spent more than a year on the Sandwich Islands observing for latitude, while at the same time observations were continued at Berlin, Prague and Strasburg, in Europe, 
and at Bethlehem, Rockville and San Francisco, in the United States. The results of all these observations have been published and show, without a chance of error, that the earth's axis is moving, that the latitudes at the Sandwich Islands increased when the latitudes in Germany diminished and vice versa.

The law of the change was eagerly and industriously sought for by some of the ablest mathematical astronomers of the world. They first worked on the idea that the changes must conform to the 305-day period of Euler, combined with an annual change due to causes set forth by Sir W. Thompson. None of these investigations gave any satisfactory formulas for the prediction of the latitude of a place. In $1891 \mathrm{Dr}$. S. C. Chandler of Cambridge, Mass., began his investigations of the problem. He remarks:

"I deliberately put aside all teaching of theory, because it seemed to me high time that the facts should be examined by a purely inductive process ; that the nugatory results of all attempts to detect the existence of the Eulerian period (of 305 days) probably arose from a defect in the theory itself; and that the entangled condition of the whole subject required that it should be examined afresh by processes unfettered by any preconceived notions whatever. The problem which I therefore proposed to myself was to see whether it would not be possible to lay the numerous ghosts in the shape of the various discordant, residual phenomena pertaining to determinations of aberration, parallaxes, latitudes and the like, which have heretofore flitted elusively about the astronomy of precision during the century; or to reduce them to some tangible form by some simple, consistent hypothesis. * * * It was thought that if this could be done, a study of the nature of the forces are thus indicated, by which the earth's rotation is influenced, might tend to a physical explanation of them."
Dr. Chandler examined a great mass of observations, new and old, and from their discussion has obtained a formula which at the present time expresses very well the changes of latitude at any place at any epoch. For his excellent and laborious work of several years, Dr. Chandler has received medals from our National Academy and the Royal Astronomical Society of London.

The result of Dr. Chandler's investigation show that the pole of the axis of rotation of the earth may be considered as revolving from west to east in a circle with a radius of about 14 feet, with an average period of 428.6 days. The center of this circle moves from west to east around the circumference of an ellipse in about a year. The pole of the axis of figure is at the center of this ellipse. Evidences of still greater complexity in the motion of the pole seem to be exhibited by Dr. Chandler's analysis. These motions combining make the actual path of the pole sometimes the arc of an ellipse, at times a circular arc and then again almost a straight line. At times the various changes conspire to give a maximum of ".33, and at others the minimum separation of few hundredths of a second of the pole of rotation from the pole of figure.

During the year 1895 Chandler's formula makes the pole move nearly parallel with our meridian. This would produce observable changes of latitude here, but none at places 90 degrees east (in Europe) or west of us. To thoroughly test the formula observations must be kept up for many years at various places on the earth's surface.

The International Geodetic Association propose the establishment of four observatories on the same parallel of latitude: in Japan, Sicily, Virginia and California. At these places it is suggested that photographic observations be kept up for many years, so that more exact data can be ob- 
tained for calculating, if possible, a more exact formula. No adequate theoretical explanation has been found, as yet, of the observed variations, though it is suspected that the annual part of the variation is due to meteorological causes, and that the other part may be caused by changes in the relative positions of portions of the earth's mass, such as movements of great masses of water and depositions of ice and snow.

A number of important problems are involved in this question of latitude variation. All the determinations of astronomy have been made on the assumption that our latitudes do not change. When the astronomer is supplied with sufficiently exact data the determination of various constants used in astronomy must be recalculated. Dr. Chandler and others have already begun the reinvestigation.

The problems so far discussed belong to pure astronomy. In the past forty years there has grown up, with a vigorous growth, a new branch of astronomy styled by some The New Astronomy. This branch deals with the beautiful and interesting investigations of the heavenly bodies made by the aid of that wonderful instrument of modern research, the spectroscope. On this occasion I will not trespass on your patience by attempting to describe to you the achievements of the new astronomy in the examination of the sun and the planets, the stars, nebulæ and comets. By the investigations of this young science of spectroscopy applied to the heavenly bodies, we get our first and accurate ideas of their constitution. On the spectroscopist we must depend for our knowledge of the surroundings of the sun and planets-the materials entering into the make-up of the stars, comets and nebulæ. The study of the stellar spectra brings wonderful information in regard to variable stars and the motions of stars.

The discoveries of argon and helium have unlocked some doors to knowledge previously closed tightly. On astrophysics the astronomer of the 20th century must depend for solving many problems. It is likely that a study of planetary spectra will give us the means of determining the rotation times of the planets-Venus and Mercury.

We have thus briefly and inadequately mentioned some of the problems which the astronomer of the next century must deal with. When we consider the progress made during the past twenty-years only, we are led to believe that world-wide coöperation in astronomical work will be one of the great features of the coming century. Only by such coöperation, directed by the ablest astronomers, can the most effective work be done. With such coöperation many of the troublesome problems will undoubtedly be solved.

J. K. REES.

\section{A NEW FORM OF RADIATION.*}

As my investigations will have to be interrupted for several weeks, I propose in the following paper to communicate a few new results.

$\S 18$. At the time of my first communication it wasknown to me that $X$-rays were able to discharge electrified bodies, and I suspected that it was X-rays, not the unaltered eathode rays, which got through his aluminum window, that Lenard had to do with in connection with distant electrified bodies. When I published my researches, however, I decided to wait until I could communicate unexceptionable results. Such are only obtainable when one makes the observation in a space which is not only completely protected against the electrostatic influences of the vacuum tube, leading-in wires, induction coil, etc., but which is also protected against the air coming from the

\footnotetext{
*Second communication to the Würzburg PhysicoMedical Society. Reprinted from the translation in Electricity.
} 